\title{
Study on Amphipathic Modification and QSAR of Volatile Turpentine Analogues as Value-added Botanical Fungicides Against Crop-threatening Pathogenic Fungi
}

\author{
Yanqing Gao, ${ }^{\dagger}$ Xiangrong Tian, ${ }^{\dagger}$ Jian Li, ${ }^{*}$ Shibin Shang, ${ }^{\#}$ Zhanqiang Song, \\ and Minggui Shen ${ }^{\#}$ \\ ${ }^{\dagger}$ College of Plant Protection, Northwest Agriculture and Forestry University, \\ Yangling, Shaanxi 712100, People's Republic of China. \\ * College of Forestry, Northwest Agriculture and Forestry University, Yangling, \\ Shaanxi 712100, People's Republic of China. \\ \# Institute of Chemical Industry of Forest Products, Chinese Academy of Forestry, \\ Nanjing, Jiangsu 210042, People's Republic of China. \\ *Corresponding author: Jian Li, ericlee99@ nwsuaf.edu.cn.
}




\section{4-isopropylcyclohexa-1, 3-dienecarboxylic acid (3, $\left.\mathrm{C}_{10} \mathrm{H}_{16} \mathrm{O}_{3}\right)$}

Yield $=70 \%$, white solid, the approximate purity was $95 \%$. IR $\left(\mathrm{cm}^{-1}\right): 3415(\mathrm{O}-\mathrm{H}) ; 2961,2874$

$\left(-\mathrm{CH}_{3},-\mathrm{CH}_{2}\right) ; 1700(-\mathrm{C}=\mathrm{O}) .{ }^{1} \mathrm{H} \mathrm{NMR}\left(\mathrm{CDCl}_{3}, \delta / \mathrm{ppm}, 300 \mathrm{MHz}\right): 6.94(\mathrm{~d}, 1 \mathrm{H}, \mathrm{O}=\mathrm{C}-\mathrm{C}=\mathrm{CH}-)$;

$5.90\left(\mathrm{~d}, 1 \mathrm{H},-\mathrm{CH}=\mathrm{C}-(\mathrm{Me})_{2}\right) ; 2.46\left(\mathrm{~m}, \mathrm{H},-\mathrm{CH}-(\mathrm{Me})_{2}\right) ; 2.51\left(\mathrm{t}, 2 \mathrm{H},-\mathrm{CH}_{2}-\mathrm{C}-\mathrm{C}=\mathrm{O}\right) ; 2.27(\mathrm{t}, 2 \mathrm{H}$,

$\left.-\mathrm{CH}_{2}-\mathrm{C}-\mathrm{C}=\mathrm{C}\right) ; 1.10\left(\mathrm{~d}, 6 \mathrm{H},-\mathrm{CH}_{3}\right)$. TOF-ESI-MS (m/z): $167[\mathrm{M}+\mathrm{H}]^{+}$. Anal. calcd for $\mathrm{C}_{10} \mathrm{H}_{14} \mathrm{O}_{2}$ :

C, 72.29; H, 11.67. Found: C, 73.11; H, 10.99.

\section{Methyl 4-isopropylcyclohexa-1, 3-dienecarboxylate $\left(5 \mathrm{a}, \mathrm{C}_{11} \mathrm{H}_{16} \mathrm{O}_{2}\right)$}

Yield $=60 \%$, yellow liquor, the approximate purity was $93 \% . \mathrm{IR}\left(\mathrm{cm}^{-1}\right): 2960,2873\left(-\mathrm{CH}_{3},-\mathrm{CH}_{2}\right)$; 1709(O-C=O); 1273, 1088(C-O-C). ${ }^{1} \mathrm{H}$ NMR $\left(\mathrm{CDCl}_{3} . \delta / \mathrm{ppm} .300 \mathrm{MHz}\right): 6.99,7.00(\mathrm{~d}, 1 \mathrm{H}$, OC-C=CH- $) ; 5.81-5.83\left(\mathrm{~d}, 1 \mathrm{H}, \mathrm{CH}=\mathrm{C}(\mathrm{Me})_{2}\right) ; 3.92\left(\mathrm{~s}, 3 \mathrm{H},-\mathrm{OCH}_{3}\right) ; 2.91-3.00\left(\mathrm{~m}, 1 \mathrm{H},-\mathrm{CH}(\mathrm{Me})_{2}\right)$; 2.41-2.48 (t, 2H, CO-C-CH $\left.\mathrm{CH}_{2}\right) ; 2.16-2.23\left(\mathrm{t}, 2 \mathrm{H}, \mathrm{CH}=\mathrm{C}-\mathrm{CH}_{2}\right) ; 1.25,1.28\left(\mathrm{~d}, 6 \mathrm{H},-2 \mathrm{CH}_{3}\right)$. TOF-ESI-MS (m/z): 179[M-H] $; 181[\mathrm{M}+\mathrm{H}]^{+}$.

\section{Ethyl 4-isopropylcyclohexa-1, 3-dienecarboxylate $\left(5 \mathrm{~b}, \mathrm{C}_{12} \mathrm{H}_{18} \mathrm{O}_{2}\right)$}

Yield $=59 \%$, yellow liquor, the approximate purity was $93 \% . \mathrm{IR}\left(\mathrm{cm}^{-1}\right): 2961,2872\left(-\mathrm{CH}_{3},-\mathrm{CH}_{2}\right)$; 1708(O-C=O); 1263, 1093(C-O-C). ${ }^{1} \mathrm{H}$ NMR(CDCl $\left.. \delta / p p m .300 M H z\right): 6.99,7.00(\mathrm{~d}, 1 \mathrm{H}$, OC-C $=\mathrm{CH}-) ; 5.80-5.82\left(\mathrm{~d}, 1 \mathrm{H},-\mathrm{CH}=\mathrm{C}-(\mathrm{Me})_{2}\right) ; 4.17-4.24\left(\mathrm{q}, 2 \mathrm{H},-\mathrm{OCH}_{2}-\right) ; 2.91-3.00(\mathrm{~m}, 1 \mathrm{H}$, $\left.-\mathrm{CH}(\mathrm{Me})_{2}\right) ; 2.41-2.48\left(\mathrm{t}, 2 \mathrm{H}, \mathrm{CO}-\mathrm{C}-\mathrm{CH}_{2}\right) ; 2.16-2.22\left(\mathrm{t}, 2 \mathrm{H}, \mathrm{CH}=\mathrm{C}-\mathrm{CH}_{2}\right) ; 1.34-1.44(\mathrm{t}, 3 \mathrm{H}$, O- $\left.\mathrm{CH}_{2}-\mathrm{CH}_{3}\right) ; 1.25,1.28\left(\mathrm{~d}, 6 \mathrm{H},-2 \mathrm{CH}_{3}\right)$. TOF-ESI-MS (m/z): 193[M-H] $; 195[\mathrm{M}+\mathrm{H}]^{+}$.

\section{Propyl 4-isopropylcyclohexa-1, 3-dienecarboxylate $\left(5 c, \mathrm{C}_{13} \mathrm{H}_{20} \mathrm{O}_{2}\right)$}

Yield $=57 \%$, yellow liquor, the approximate purity was $90 \%$. IR $\left(\mathrm{cm}^{-1}\right): 2962,2876\left(-\mathrm{CH}_{3},-\mathrm{CH}_{2}\right)$; $1704(\mathrm{O}-\mathrm{C}=\mathrm{O}) ; 1260,1083$ (C-O-C). ${ }^{1} \mathrm{H}$ NMR $\left(\mathrm{CDCl}_{3} . \delta / \mathrm{ppm} .300 \mathrm{MHz}\right)$ : 6.99-7.01 (d, 1H, $\mathrm{OC}-\mathrm{C}=\mathrm{CH}-) ; 5.81-5.83\left(\mathrm{~d}, 1 \mathrm{H}, \mathrm{CH}=\mathrm{C}(\mathrm{Me})_{2}\right) ; 4.09-4.13\left(\mathrm{t}, 2 \mathrm{H}, \mathrm{O}-\mathrm{CH}_{2}-\right) ; 2.90-2.98(\mathrm{~m}, 1 \mathrm{H}$, $\left.-\mathrm{CH}(\mathrm{Me})_{2}\right) ; 2.42-2.48\left(\mathrm{t}, 2 \mathrm{H}, \mathrm{CO}-\mathrm{C}-\mathrm{CH}_{2}\right) ; 2.17-2.23\left(\mathrm{t}, 2 \mathrm{H}, \mathrm{CH}=\mathrm{C}-\mathrm{CH}_{2}\right) ; 1.63-1.82(\mathrm{~m}, 2 \mathrm{H}$, $\left.-\mathrm{CH}_{2}-\right)$;1.04-1.08 (d, 6H, -2 $\left.\mathrm{CH}_{3}\right)$; 0.94-0.99 (t, 3H, $\left.-\mathrm{CH}_{3}\right)$. TOF-ESI-MS (m/z): 209[M+H] $]^{+}$

\section{Isopropyl 4-isopropylcyclohexa-1, 3-dienecarboxylate $\left(5 \mathrm{~d}, \mathrm{C}_{13} \mathrm{H}_{20} \mathrm{O}_{2}\right)$}


Yield $=60 \%$, yellow liquor, the approximate purity was $90 \%$. IR $\left(\mathrm{cm}^{-1}\right): 2963,2873\left(-\mathrm{CH}_{3},-\mathrm{CH}_{2}\right)$; $1703(\mathrm{O}-\mathrm{C}=\mathrm{O}) ; 1384,1373\left(\mathrm{CH}(\mathrm{Me})_{2}\right) ; 1255,1108(\mathrm{C}-\mathrm{O}-\mathrm{C})$.

${ }^{1} \mathrm{H}$ NMR $\left(\mathrm{CDCl}_{3}\right.$. $\delta / \mathrm{ppm}$. 300MHz): 6.97-6.99 (d, 1H, OC-C=CH-); 5.80-5.82 (d, $1 \mathrm{H}, \mathrm{CH}=$ $\mathrm{C}(\mathrm{Me})_{2} ;$ 5.12-5.51 (m, 1H, O-CH-); 2.88-2.98 (m, 1H, -CH(Me) $)$ ); 2.41-2.47 (t, 2H, CO-C-CH ${ }_{2}$; 2.16-2.22 (t, $\left.2 \mathrm{H}, \mathrm{CH}=\mathrm{C}-\mathrm{CH}_{2}\right) ; 1.35,1.37\left(\mathrm{~d}, 6 \mathrm{H},-2 \mathrm{CH}_{3}\right) ; 1.26,1.28\left(\mathrm{~d}, 6 \mathrm{H},-2 \mathrm{CH}_{3}\right)$. TOF-ESI-MS $(\mathrm{m} / \mathrm{z}): 209[\mathrm{M}+\mathrm{H}]^{+}$.

\section{Butyl 4-isopropylcyclohexa-1,3-dienecarboxylate $\left(5 e, \mathrm{C}_{14} \mathrm{H}_{22} \mathrm{O}_{2}\right)$}

Yield $=60 \%$, yellow liquor, the approximate purity was $90 \%$. IR $\left(\mathrm{cm}^{-1}\right): 2959,2872\left(-\mathrm{CH}_{3},-\mathrm{CH}_{2}\right)$; 1708(O-C=O); 1251, 1082 (C-O-C). ${ }^{1} \mathrm{H}$ NMR $\left(\mathrm{CDCl}_{3} . \delta / \mathrm{ppm} .300 \mathrm{MHz}\right): 6.98-7.00(\mathrm{~d}, 1 \mathrm{H}$, OC-C=CH- $)$ 5.81-5.83 (d, $\left.1 \mathrm{H}, \mathrm{CH}=\mathrm{C}(\mathrm{Me})_{2}\right) ; 4.12-4.17\left(\mathrm{t}, 2 \mathrm{H}, \mathrm{O}-\mathrm{CH}_{2}\right) ; 2.89-2.98(\mathrm{~m}, 1 \mathrm{H}$, $\left.-\mathrm{CH}(\mathrm{Me})_{2}\right) ; 2.44-2.48\left(\mathrm{t}, 2 \mathrm{H}, \mathrm{CO}-\mathrm{C}-\mathrm{CH}_{2}\right) ; 2.16-2.22\left(\mathrm{t}, 2 \mathrm{H}, \mathrm{CH}=\mathrm{C}-\mathrm{CH}_{2}\right) ; 1.60-1.67(\mathrm{~m}, 2 \mathrm{H}$, O- $\left.\mathrm{CH}_{2}-\mathrm{CH}_{2}-\right) ; 1.35-1.45\left(\mathrm{~m}, 2 \mathrm{H}, \mathrm{O}-\mathrm{CH}_{2}-\mathrm{CH}_{2}-\mathrm{CH}_{2}\right) ; 1.05-1.07$ (d, 6H, $\left.-2 \mathrm{CH}_{3}\right) ; 0.92-0.97$ (t, $3 \mathrm{H}$, $\left.-\mathrm{CH}_{3}\right)$. TOF-ESI-MS (m/z): 223[M+H] $; 246[\mathrm{M}+\mathrm{Na}]^{+}$.

Isobutyl 4-isopropylcyclohexa-1,3-dienecarboxylate $\left(5 \mathrm{f}, \mathrm{C}_{14} \mathrm{H}_{22} \mathrm{O}_{2}\right)$

Yield $=60 \%$, yellow liquor, the approximate purity was $90 \%$. IR $\left(\mathrm{cm}^{-1}\right): 2960,2873\left(-\mathrm{CH}_{3},-\mathrm{CH}_{2}\right)$; 1709(O-C=O); 1191, 1167(-CH(Me) $\left.)_{2}\right) ; 1080,1248(\mathrm{C}-\mathrm{O}-\mathrm{C})$.

${ }^{1} \mathrm{H}$ NMR $\left(\mathrm{CDCl}_{3}\right.$. $\left.\delta / \mathrm{ppm} .300 \mathrm{MHz}\right)$ : 7.00-7.01 (d, 1H, OC-C=CH-); 5.81-5.83 (d, 1H, CH= $\left.\mathrm{C}(\mathrm{Me})_{2}\right) ; 3.92-3.94\left(\mathrm{~d}, 2 \mathrm{H}, \mathrm{O}_{-} \mathrm{CH}_{2}-\right) ; 2.90-2.96\left(\mathrm{~m}, 1 \mathrm{H},-\mathrm{CH}(\mathrm{Me})_{2}\right) ; 2.82-2.85\left(\mathrm{~m}, 1 \mathrm{H},-\mathrm{CH}(\mathrm{Me})_{2}\right)$; 2.42-2.49 (t, $\left.2 \mathrm{H}, \mathrm{CO}-\mathrm{C}-\mathrm{CH}_{2}\right) ; 2.17-2.23\left(\mathrm{t}, 2 \mathrm{H}, \mathrm{CH}=\mathrm{C}-\mathrm{CH}_{2}\right) ; 1.02-1.08\left(\mathrm{~d}, 6 \mathrm{H},-2 \mathrm{CH}_{3}\right)$, 0.95-0.97 (d, 6H, -2 $\left.\mathrm{CH}_{3}\right)$. TOF-ESI-MS (m/z): 223[M+H] $]^{+} ; 246[\mathrm{M}+\mathrm{Na}]^{+}$.

\section{Tert-butyl 4-isopropylcyclohexa-1,3-dienecarboxylate $\left(5 \mathrm{~g}, \mathrm{C}_{14} \mathrm{H}_{22} \mathrm{O}_{2}\right)$}

Yield $=55 \%$, yellow liquor, the approximate purity was $91 \%$. IR $\left(\mathrm{cm}^{-1}\right): 2963,2872\left(-\mathrm{CH}_{3},-\mathrm{CH}_{2}\right)$; 1702(O-C=O); 1390, 1367(C(Me) $)$ ); 1085, 1275 (C-O-C).

${ }^{1} \mathrm{H}$ NMR $\left(\mathrm{CDCl}_{3}\right.$. $\left.\delta / \mathrm{ppm} .300 \mathrm{MHz}\right): 6.93-6.91(\mathrm{~d}, 1 \mathrm{H}, \mathrm{OC}-\mathrm{C}=\mathrm{CH}-) ; 5.81-5.79(\mathrm{~d}, 1 \mathrm{H}, \mathrm{CH}=$ $\left.\mathrm{C}(\mathrm{Me})_{2}\right) ; 2.85-2.97\left(\mathrm{~m}, 1 \mathrm{H},-\mathrm{CH}(\mathrm{Me})_{2}\right) ; 2.37-2.43\left(\mathrm{t}, 2 \mathrm{H}, \mathrm{CO}-\mathrm{C}_{-} \mathrm{CH}_{2}\right) ; 2.15-2.21(\mathrm{t}, 2 \mathrm{H}$, $\left.\mathrm{CH}=\mathrm{C}-\mathrm{CH}_{2}\right) ; 1.49\left(\mathrm{~s}, 9 \mathrm{H}, 3-\mathrm{CH}_{3}\right) ; 1.01-1.07\left(\mathrm{~d}, 6 \mathrm{H},-2 \mathrm{CH}_{3}\right)$. TOF-ESI-MS (m/z): 223[M+H] ${ }^{+}$, $246[\mathrm{M}+\mathrm{Na}]^{+}$.

Sec-butyl 4-isopropylcyclohexa-1,3-dienecarboxylate $\left(5 \mathrm{~h}, \mathrm{C}_{14} \mathrm{H}_{22} \mathrm{O}_{2}\right)$ 
Yield $=55 \%$, yellow liquor, the approximate purity was $91 \%$. IR $\left(\mathrm{cm}^{-1}\right): 2963,2872\left(-\mathrm{CH}_{3},-\mathrm{CH}_{2}\right)$; 1702(O-C=O); 1085, 1275 (C-O-C). ${ }^{1} \mathrm{H}$ NMR ( $\left.\mathrm{CDCl}_{3} . \delta / \mathrm{ppm} .300 \mathrm{MHz}\right): 6.93-6.91(\mathrm{~d}, 1 \mathrm{H}$, OC-C=CH-); 5.81-5.79 (d, $\left.1 \mathrm{H}, \mathrm{CH}=\mathrm{C}(\mathrm{Me})_{2}\right) ; 4.13(\mathrm{~m}, 1 \mathrm{H}, \mathrm{COO}-\mathrm{CH}-) ; 2.85-2.97(\mathrm{~m}, 1 \mathrm{H}$, $\left.-\mathrm{CH}(\mathrm{Me})_{2}\right) ; 2.15-2.21\left(\mathrm{t}, 2 \mathrm{H}, \mathrm{CH}=\mathrm{C}-\mathrm{CH}_{2}\right) ; 1.57\left(\mathrm{~m}, 2 \mathrm{H},-\mathrm{CH}_{2}-\right) ; 1.40\left(\mathrm{~d}, 3 \mathrm{H},-\mathrm{CH}_{3}\right), 1.01-1.07$ (d, $\left.6 \mathrm{H},-2 \mathrm{CH}_{3}\right), 0.96\left(\mathrm{t}, 3 \mathrm{H},-\mathrm{CH}_{3}\right)$. TOF-ESI-MS (m/z): 223[M+H] ${ }^{+} ; 246[\mathrm{M}+\mathrm{Na}]^{+}$.

\section{Pentyl 4-isopropylcyclohexa-1,3-dienecarboxylate $\left(5 \mathrm{i}, \mathrm{C}_{15} \mathrm{H}_{24} \mathrm{O}_{2}\right)$}

Yield $=57 \%$, yellow liquor, the approximate purity was $91 \%$. IR $\left(\mathrm{cm}^{-1}\right): 2963,2872\left(-\mathrm{CH}_{3},-\mathrm{CH}_{2}\right)$; 1702(O-C=O); 1085,1275 (C-O-C). ${ }^{1} \mathrm{H}$ NMR ( $\left.\mathrm{CDCl}_{3} . \delta / \mathrm{ppm} .300 \mathrm{MHz}\right): 6.93-6.91$ (d, 1H, OC-C=CH- $)$ 5.81-5.79 (d, $\left.1 \mathrm{H}, \mathrm{CH}=\mathrm{C}(\mathrm{Me})_{2}\right) ; 4.15\left(\mathrm{t}, 2 \mathrm{H}, \mathrm{COO}^{\left.-\mathrm{CH}_{2}-\right)} ; 2.85-2.97(\mathrm{~m}, 1 \mathrm{H}\right.$, $\left.-\mathrm{CH}(\mathrm{Me})_{2}\right) ; 2.15-2.21\left(\mathrm{t}, 2 \mathrm{H}, \mathrm{CH}=\mathrm{C}-\mathrm{CH}_{2}\right) ; 1.57\left(\mathrm{~m}, 2 \mathrm{H},-\mathrm{CH}_{2}-\right) ; 1.29-1.33\left(\mathrm{~m}, 4 \mathrm{H},-\mathrm{CH}_{2}-\right)$; 1.01-1.07 (d, 6H, -2 $\left.\mathrm{CH}_{3}\right), 0.96\left(\mathrm{t}, 3 \mathrm{H},-\mathrm{CH}_{3}\right)$. TOF-ESI-MS (m/z): 237[M+H] $]^{+}$

\section{Cyclohexyl 4-isopropylcyclohexa-1,3-dienecarboxylate $\left(5 \mathrm{j}, \mathrm{C}_{16} \mathrm{H}_{24} \mathrm{O}_{2}\right)$}

Yield $=58 \%$, yellow liquor, the approximate purity was $90 \%$. IR $\left(\mathrm{cm}^{-1}\right): 2963,2872\left(-\mathrm{CH}_{3},-\mathrm{CH}_{2}\right)$; 1702(O-C=O); 1085, 1275 (C-O-C). ${ }^{1} \mathrm{H}$ NMR $\left(\mathrm{CDCl}_{3} . \delta / \mathrm{ppm} .300 \mathrm{MHz}\right): 6.93-6.91(\mathrm{~d}, 1 \mathrm{H}$, OC-C=CH- $)$ 5.81-5.79 (d, $\left.1 \mathrm{H}, \mathrm{CH}=\mathrm{C}(\mathrm{Me})_{2}\right) ; 3.91(\mathrm{~m}, 1 \mathrm{H}, \mathrm{COO}-\mathrm{CH}-) ; 2.85-2.97(\mathrm{~m}, 1 \mathrm{H}$, $\left.-\mathrm{CH}(\mathrm{Me})_{2}\right) ; 2.15-2.21\left(\mathrm{t}, 2 \mathrm{H}, \mathrm{CH}=\mathrm{C}-\mathrm{CH}_{2}\right) ; 1.39-1.80\left(\mathrm{~m}, 10 \mathrm{H},-\mathrm{CH}_{2^{-}}\right) ; 1.01-1.07(\mathrm{~d}, 6 \mathrm{H}$, $\left.-2 \mathrm{CH}_{3}\right)$.TOF-ESI-MS (m/z): 249[M+H] $]^{+}$

\section{2-hydroxyethyl 4-isopropylcyclohexa-1,3-dienecarboxylate (5k, $\left.\mathrm{C}_{12} \mathrm{H}_{18} \mathrm{O}_{3}\right)$}

Yield $=55 \%$, yellow liquor, the approximate purity was $90 \%$. IR $\left(\mathrm{cm}^{-1}\right): 3010(-\mathrm{OH}) ; 2963$, $2872\left(-\mathrm{CH}_{3},-\mathrm{CH}_{2}\right) ; 1702(\mathrm{O}-\mathrm{C}=\mathrm{O}) ; 1085,1275$ (C-O-C). ${ }^{1} \mathrm{H}$ NMR $\left(\mathrm{CDCl}_{3} . \delta / \mathrm{ppm} .300 \mathrm{MHz}\right)$ : 6.93-6.91 (d, 1H, OC-C=CH-); 5.81-5.79 (d, 1H, CH=C(Me $\left.)_{2}\right) ; 4.34\left(\mathrm{t}, 2 \mathrm{H}, \mathrm{COO}-\mathrm{CH}_{2^{-}}\right) ; 3.81(\mathrm{t}$, $\left.2 \mathrm{H},-\mathrm{CH}_{2}-\mathrm{OH}\right) ; 2.85-2.97\left(\mathrm{~m}, 1 \mathrm{H},-\mathrm{CH}(\mathrm{Me})_{2}\right) ; 2.15-2.21\left(\mathrm{t}, 2 \mathrm{H}, \mathrm{CH}=\mathrm{C}-\mathrm{CH}_{2}\right) ; 1.01-1.07(\mathrm{~d}, 6 \mathrm{H}$, $\left.-2 \mathrm{CH}_{3}\right)$. TOF-ESI-MS (m/z): 211[M+H $]^{+}$.

\section{2-(2-hydroxyethoxy)ethyl 4-isopropylcyclohexa-1,3-dienecarboxylate $\left(51, \mathrm{C}_{14} \mathrm{H}_{22} \mathrm{O}_{4}\right)$}

Yield $=55 \%$, yellow liquor, the approximate purity was $90 \%$. IR $\left(\mathrm{cm}^{-1}\right): 3013(-\mathrm{OH}) ; 2963,2872$ $\left(-\mathrm{CH}_{3},-\mathrm{CH}_{2}\right) ; 1702(\mathrm{O}-\mathrm{C}=\mathrm{O}) ; 1085,1275$ (C-O-C). ${ }^{1} \mathrm{H}$ NMR $\left(\mathrm{CDCl}_{3} . \quad \delta / \mathrm{ppm} . \quad 300 \mathrm{MHz}\right)$ : 6.93-6.91(d, 1H, OC-C=CH-); 5.81-5.79 (d, 1H, CH=C(Me $\left.)_{2}\right) ; 4.32\left(\mathrm{t}, 2 \mathrm{H},-\mathrm{COO}_{-} \mathrm{CH}_{2^{-}}\right) ; 3.70$ (t, 
$2 \mathrm{H},-\mathrm{CH}_{2}-\mathrm{OH}$ ); 3.65-3.56 (t, 4H, - $\left.\mathrm{CH}_{2}-\mathrm{O}-\right)$; 2.85-2.97 (m, $\left.1 \mathrm{H},-\mathrm{CH}(\mathrm{Me}) 2\right) ; 2.15-2.21$ (t, $2 \mathrm{H}$, $\left.\mathrm{CH}=\mathrm{C}-\mathrm{CH}_{2}\right) ; 1.01-1.07\left(\mathrm{~d}, 6 \mathrm{H},-2 \mathrm{CH}_{3}\right)$. TOF-ESI-MS (m/z): 255[M+H] $]^{+}$

2-ethyl di [4-isopropylcyclohexa-1,3-dienecarboxylate] $\left(5 \mathrm{~m}, \mathrm{C}_{22} \mathrm{H}_{30} \mathrm{O}_{4}\right)$

Yield $=50 \%$, yellow liquor, the approximate purity was $88 \%$. IR $\left(\mathrm{cm}^{-1}\right): 2963,2872\left(-\mathrm{CH}_{3},-\mathrm{CH}_{2}\right)$; 1702 (O-C=O); 1085, 1275 (C-O-C). ${ }^{1} \mathrm{H}$ NMR $\left(\mathrm{CDCl}_{3} . \delta / \mathrm{ppm} .300 \mathrm{MHz}\right): 6.93-6.91(\mathrm{~d}, 1 \mathrm{H}$,

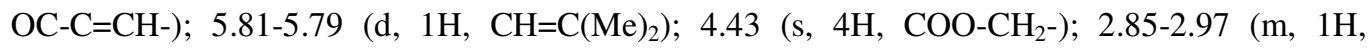
$\left.-\mathrm{CH}(\mathrm{Me})_{2}\right) ; 2.15-2.21\left(\mathrm{t}, 2 \mathrm{H}, \mathrm{CH}=\mathrm{C}-\mathrm{CH}_{2}\right) ; 1.01-1.07\left(\mathrm{~d}, 6 \mathrm{H},-2 \mathrm{CH}_{3}\right)$. TOF-ESI-MS (m/z): $363[\mathrm{M}+\mathrm{H}]^{+}$.

\section{2-(2-hydroxyethoxy)ethy di [4-isopropylcyclohexa-1,3-dienecarboxylate] $\left(5 n, \mathrm{C}_{25} \mathrm{H}_{34} \mathrm{O}_{4}\right)$}

Yield $=50 \%$, yellow liquor, the approximate purity was $85 \%$. IR $\left(\mathrm{cm}^{-1}\right): 2963,2872\left(-\mathrm{CH}_{3},-\mathrm{CH}_{2}\right)$; 1702 (O-C=O); 1085, 1275 (C-O-C). ${ }^{1} \mathrm{H}$ NMR ( $\left.\mathrm{CDCl}_{3} . \delta / \mathrm{ppm} .300 \mathrm{MHz}\right): 6.93-6.91$ (d, 1H, OC-C=CH-); 5.81-5.79 (d, 1H, CH=C(Me $\left.)_{2}\right) ; 4.32\left(\mathrm{t}, 4 \mathrm{H},-\mathrm{COO}^{-\mathrm{CH}_{2}-}\right) ; 3.65$ (t, 4H, $\left.-\mathrm{CH}_{2}-\mathrm{O}-\right)$; 2.85-2.97 (m, $\left.1 \mathrm{H},-\mathrm{CH}(\mathrm{Me})_{2}\right) ; 2.15-2.21\left(\mathrm{t}, 2 \mathrm{H}, \mathrm{CH}=\mathrm{C}-\mathrm{CH}_{2}\right) ; 1.01-1.07\left(\mathrm{~d}, 6 \mathrm{H},-2 \mathrm{CH}_{3}\right)$. TOF-ESI-MS (m/z): 403[M+H] $]^{+}$

\section{Isopropyl- $N^{2}, N^{3}, 4$-trimethylbicyclo[2.2.2]oct-5-ene-2,3-dicarboxamide (7a, $\left.\mathrm{C}_{16} \mathrm{H}_{26} \mathrm{~N}_{2} \mathrm{O}_{2}\right)$}

Yield $=50 \%$, yellow solid, the approximate purity was $88 \%$. IR $\left(\mathrm{cm}^{-1}\right): 3400\left(-\mathrm{NH}_{2}\right)$; 1668( $\left.\mathrm{H}_{2} \mathrm{~N}-\mathrm{C}=\mathrm{O}\right) .{ }^{1} \mathrm{H}$ NMR $\left(\mathrm{CDCl}_{3} . \delta / \mathrm{ppm} .300 \mathrm{MHz}\right): 6.0$ (s, 4H, - $\left.\mathrm{NH}_{2}\right) ; 5.60$ (d, 2H, - $\left.\mathrm{CH}=\mathrm{CH}-\right)$; 2.62 (s, 2H, -CH-); 1.82-1.87(m, 1H, -CH-); 1.50, 1.30 (t, 4H, - $\left.\mathrm{CH}_{2}-\right) ; 1.20$ (s, 3H, $\left.-\mathrm{CH}_{3}\right) ; 1.00$, 1.01(d, 6H, - $\left.\mathrm{CH}_{3}\right)$.TOF-ESI-MS (m/z): 251[M+H] ${ }^{+} ; 274[\mathrm{M}+\mathrm{Na}]^{+}$.

\section{$\mathrm{C}_{18} \mathrm{H}_{30} \mathrm{~N}_{2} \mathrm{O}_{2}$ )}

Yield $=48 \%$, yellow solid, the approximate purity was $88 \%$. IR $\left(\mathrm{cm}^{-1}\right): 3400(-\mathrm{NH}-)$; 1668(-HN-C=O). ${ }^{1} \mathrm{H}$ NMR $\left(\mathrm{CDCl}_{3} . \delta / \mathrm{ppm} .300 \mathrm{MHz}\right): 8.0$ (q, 2H, -NH-); 5.60 (d, 2H, -CH=CH-); 2.67 (s, 6H, -N-CH 3 ); 2.62 (s, 2H, -CH-); 1.82-1.87 (m, 1H, -CH-); 1.50, 1.30 (t, 4H, - $\mathrm{CH}_{2}$-); 1.20 (s, 3H, - $\left.\mathrm{CH}_{3}\right) ; 1.00,1.01\left(\mathrm{~d}, 6 \mathrm{H},-\mathrm{CH}_{3}\right)$. TOF-ESI-MS (m/z): $279[\mathrm{M}+\mathrm{H}]^{+} ; 302[\mathrm{M}+\mathrm{Na}]^{+}$. 
Yield $=44 \%$, yellow solid, the approximate purity was $85 \%$. IR $\left(\mathrm{cm}^{-1}\right): 3400(-\mathrm{NH}-)$; 1668(-HN-C=O). ${ }^{1} \mathrm{H}$ NMR ( $\left.\mathrm{CDCl}_{3} . \delta / \mathrm{ppm} .300 \mathrm{MHz}\right): 8.0$ (q, 2H, -NH-); 5.60 (d, 2H, -CH=CH-); 3.27 (q, 4H, -N-CH $\left.2^{-}\right) ; 2.62$ (s, 2H, -CH-); 1.82-1.87 (m, 1H, -CH-); 1.50, 1.30 (t, 4H, $-\mathrm{CH}_{2}$ ); $1.25\left(\mathrm{t}, 6 \mathrm{H},-\mathrm{CH}_{3}\right) ; 1.20\left(\mathrm{~s}, 3 \mathrm{H},-\mathrm{CH}_{3}\right) ; 1.00,1.01\left(\mathrm{~d}, 6 \mathrm{H},-\mathrm{CH}_{3}\right)$. TOF-ESI-MS $(\mathrm{m} / \mathrm{z}): 307$ $[\mathrm{M}+\mathrm{H}]^{+}$.

$N^{2}, N^{3}$,1-triisopropyl-4-methylbicyclo[2.2.2] $]$ oct-5-ene-2,3-dicarboxamide (7d, $\left.\mathrm{C}_{20} \mathrm{H}_{34} \mathrm{~N}_{2} \mathrm{O}_{2}\right)$ Yield $=45 \%$, yellow solid, the approximate purity was $85 \%$. IR $\left(\mathrm{cm}^{-1}\right): 3400(-\mathrm{NH}-)$; 1668(-HN-C=O). ${ }^{1} \mathrm{H}$ NMR ( $\left.\mathrm{CDCl}_{3} .8 / \mathrm{ppm} .300 \mathrm{MHz}\right): 8.0$ (q, 2H, -NH-); 5.60 (d, 2H, -CH=CH-); 3.27 (t, 4H, -N-CH $\left.2^{-}\right) ; 2.62$ (s, 2H, -CH-); 1.82-1.87 (m, 1H, -CH-); 1.50, 1.30 (t, 4H, - $\mathrm{CH}_{2}-$ ); 1.45 (m, 4H, - $\left.\mathrm{CH}_{2}-\right) ; 1.20\left(\mathrm{~s}, 3 \mathrm{H},-\mathrm{CH}_{3}\right) ; 1.00,1.01\left(\mathrm{~d}, 6 \mathrm{H},-\mathrm{CH}_{3}\right) ; 0.96\left(\mathrm{t}, 6 \mathrm{H},-\mathrm{CH}_{3}\right)$. TOF-ESI-MS $(\mathrm{m} / \mathrm{z}): 335[\mathrm{M}+\mathrm{H}]^{+}$.

Dimethyl 1-isopropyl-4-methylbicyclo[2.2.2] oct-5-ene-2,3-dicarboxylate $\left(8 \mathrm{a}, \mathrm{C}_{16} \mathrm{H}_{24} \mathrm{O}_{4}\right)$ :

Yield $=45 \%$, yellow liquor, the approximate purity was $88 \%$. IR $\left(\mathrm{cm}^{-1}\right): 1774,1746(\mathrm{C}=\mathrm{O}) .{ }^{1} \mathrm{H}$ NMR (CDCl $3 . \delta / p p m .300 \mathrm{MHz}): 6.00(\mathrm{~d}, 1 \mathrm{H},-\mathrm{CH}=\mathrm{CH}-) ; 6.10$ (d, 1H, -CH=CH-); 3.67 (s, 6H, $\left.-\mathrm{O}-\mathrm{CH}_{3}\right) ; 3.24$ (s, $\left.1 \mathrm{H},-\mathrm{CH}-\right) ; 3.23$ (s, $\left.1 \mathrm{H},-\mathrm{CH}-\right) ; 1.49,1.63\left(\mathrm{~d}, 4 \mathrm{H},-\mathrm{CH}_{2^{-}}\right) ; 1.81-1.87(\mathrm{~m}, 1 \mathrm{H}$, $-\mathrm{CH}-) ; \quad 1.13\left(\mathrm{~s}, \quad 3 \mathrm{H}, \quad-\mathrm{CH}_{3}\right) ; \quad 1.00, \quad 1.01\left(\mathrm{~d}, \quad 6 \mathrm{H}, \quad-\mathrm{CH}_{3}\right) ; \quad$ TOF-ESI-MS $\quad(\mathrm{m} / \mathrm{z}): 281[\mathrm{M}+\mathrm{H}]^{+}$; $303[\mathrm{M}+\mathrm{Na}]^{+}$.

Diethyl 1-isopropyl-4-methylbicyclo[2.2.2]oct-5-ene-2,3-dicarboxylate (8b, $\left.\mathrm{C}_{18} \mathrm{H}_{28} \mathrm{O}_{4}\right)$ :

Yield $=44 \%$, yellow liquor, the approximate purity was $88 \%$. IR $\left(\mathrm{cm}^{-1}\right): 1774,1746(\mathrm{C}=\mathrm{O}) .{ }^{1} \mathrm{H}$ NMR (CDCl $. \delta /$ ppm. $300 \mathrm{MHz})$ : 6.00 (d, 1H, -CH=CH-); 6.10 (d, 1H, -CH=CH-); 4.10-4.12 (m, $\left.4 \mathrm{H},-\mathrm{CH}_{2^{-}}\right) ; 3.23(\mathrm{~s}, 2 \mathrm{H},-\mathrm{CH}-) ; 1.49,1.63\left(\mathrm{~d}, 4 \mathrm{H},-\mathrm{CH}_{2^{-}}\right) ; 1.30-1.25\left(\mathrm{t}, 6 \mathrm{H},-\mathrm{CH}_{3}\right) ; 1.81-1.87(\mathrm{~m}$, $1 \mathrm{H},-\mathrm{CH}-) ; 1.13\left(\mathrm{~s}, 3 \mathrm{H},-\mathrm{CH}_{3}\right) ; 1.00,1.01\left(\mathrm{~d}, 6 \mathrm{H},-\mathrm{CH}_{3}\right)$.

TOF-ESI-MS (m/z): 295[M+H] $]^{+} ; 317[\mathrm{M}+\mathrm{Na}]^{+}$.

Dipropyl 1-isopropyl-4-methylbicyclo[2.2.2] oct-5-ene-2,3-dicarboxylate $\left(8 \mathrm{c}, \mathrm{C}_{20} \mathrm{H}_{32} \mathrm{O}_{4}\right)$ :

Yield $=45 \%$, yellow liquor, the approximate purity was $88 \%$. IR $\left(\mathrm{cm}^{-1}\right): 1774,1746(\mathrm{C}=\mathrm{O}) .{ }^{1} \mathrm{H}$ NMR (CDCl3. $\delta / p p m .300$ MHz): 6.00 (d, 1H, -CH=CH-); 6.10 (d, 1H, -CH=CH-); 4.07-4.12 (m, 4H, -O- $\left.\mathrm{CH}_{2}-\right) ; 3.23$ (s, 2H, -CH-); 1.49, 1.63 (d, 4H, - $\left.\mathrm{CH}_{2}-\right) ; 1.30-1.25\left(\mathrm{t}, 6 \mathrm{H},-\mathrm{CH}_{3}\right) ; 1.81-1.87(\mathrm{~m}$, $1 \mathrm{H},-\mathrm{CH}-) ; 1.13(\mathrm{~s}, 3 \mathrm{H},-\mathrm{CH} 3) ; 1.00,1.01\left(\mathrm{~d}, 6 \mathrm{H},-\mathrm{CH}_{3}\right)$. 
TOF-ESI-MS (m/z): 309[M+H $]^{+} ; 331[\mathrm{M}+\mathrm{Na}]^{+}$.

Diisopropyl 1-isopropyl-4-methylbicyclo[2.2.2] 0 ct-5-ene-2,3-dicarboxylate (8d, $\left.\mathrm{C}_{20} \mathrm{H}_{32} \mathrm{O}_{4}\right)$ :

Yield $=45 \%$, yellow liquor, the approximate purity was $88 \%$. IR $\left(\mathrm{cm}^{-1}\right): 1774,1746(\mathrm{C}=\mathrm{O}) .{ }^{1} \mathrm{H}$ NMR (CDCl ${ }_{3} . \delta / p p m .300$ MHz): 6.00 (d, 1H, -CH=CH-); 6.10 (d, 1H, -CH=CH-); 4.12-4.20 (m, $2 \mathrm{H},-\mathrm{O}-\mathrm{CH}-) ; 3.23$ (s, 2H, -CH-); 1.49, $1.63\left(\mathrm{~d}, 4 \mathrm{H},-\mathrm{CH}_{2}-\right) ; 1.30\left(\mathrm{~s}, 3 \mathrm{H},-\mathrm{CH}_{3}\right) ; 1.81-1.87(\mathrm{~m}, 1 \mathrm{H}$, $-\mathrm{CH}-) ; 1.35-1.40\left(\mathrm{~d}, 12 \mathrm{H},-\mathrm{CH}_{3}\right) ; 1.00,1.01\left(\mathrm{~d}, 6 \mathrm{H},-\mathrm{CH}_{3}\right)$.

TOF-ESI-MS (m/z): 309[M+H] $]^{+} ; 331[\mathrm{M}+\mathrm{Na}]^{+}$.

Dibutyl 1-isopropyl-4-methylbicyclo[2.2.2] oct-5-ene-2,3-dicarboxylate $\left(8 \mathrm{e}, \mathrm{C}_{22} \mathrm{H}_{36} \mathrm{O}_{4}\right)$ :

Yield $=45 \%$, yellow liquor, the approximate purity was $88 \%$. IR $\left(\mathrm{cm}^{-1}\right): 1774,1746(\mathrm{C}=\mathrm{O}) .{ }^{1} \mathrm{H}$

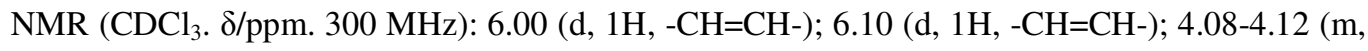
$\left.4 \mathrm{H},-\mathrm{O}-\mathrm{CH}_{2}-\right) ; 3.23$ (s, 2H, -CH-); 1.49, 1.63 (d, 4H, - $\left.\mathrm{CH}_{2^{-}}\right) ; 1.81-1.87(\mathrm{~m}, 1 \mathrm{H},-\mathrm{CH}-)$; 1.57-1.60(m, $\left.4 \mathrm{H},-\mathrm{CH}_{2^{-}}\right) ; 1.33-1.30\left(\mathrm{~m}, 4 \mathrm{H},-\mathrm{CH}_{2^{-}}\right) ; 1.32\left(\mathrm{~s}, 3 \mathrm{H},-\mathrm{CH}_{3}\right), 1.00,1.01\left(\mathrm{~d}, 6 \mathrm{H},-\mathrm{CH}_{3}\right)$; 0.90-1.00((t, 6H, - $\left.\mathrm{CH}_{3}\right)$. TOF-ESI-MS (m/z): 309[M+H] $]^{+} ; 331[\mathrm{M}+\mathrm{Na}]^{+}$. 


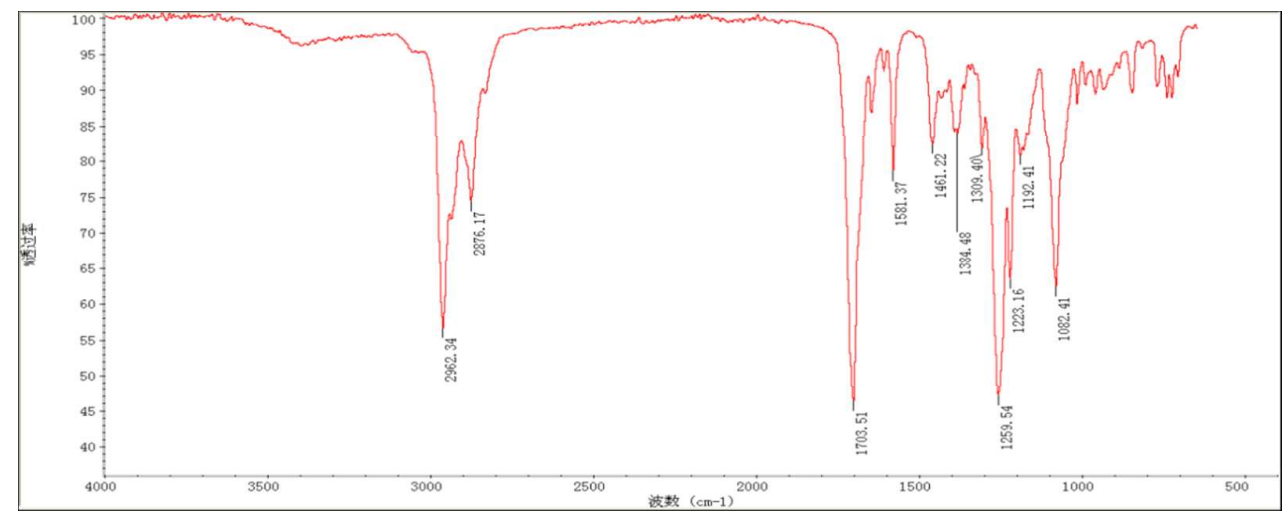

Figure S1. FT-IR spectrum of the compound 5a

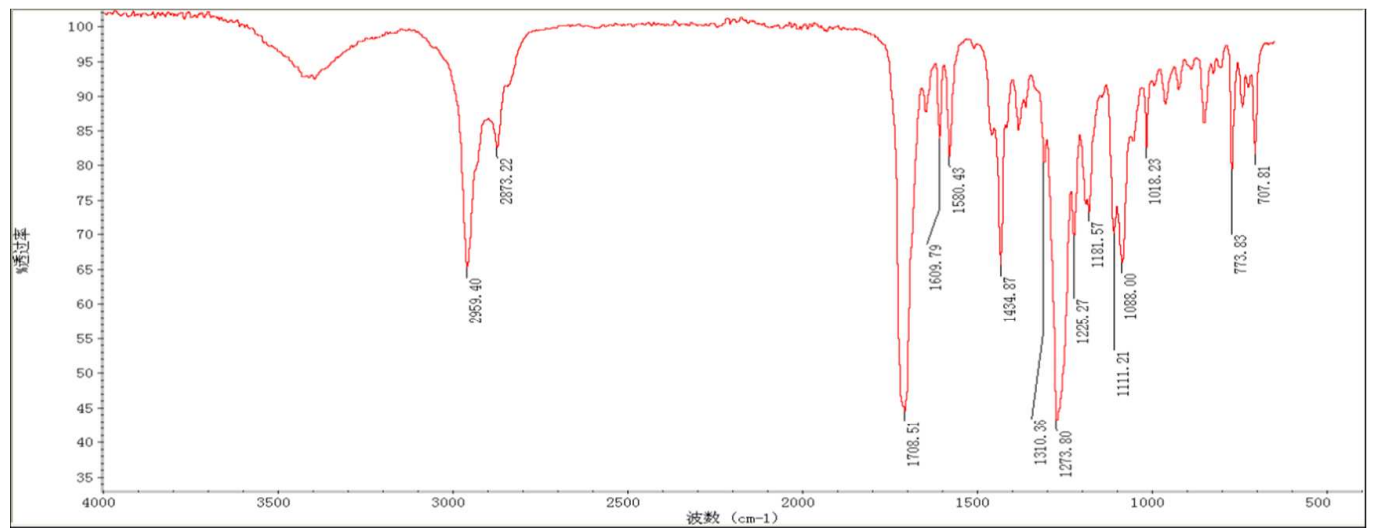

Figure S2. FT-IR spectrum of the compound $\mathbf{5 b}$

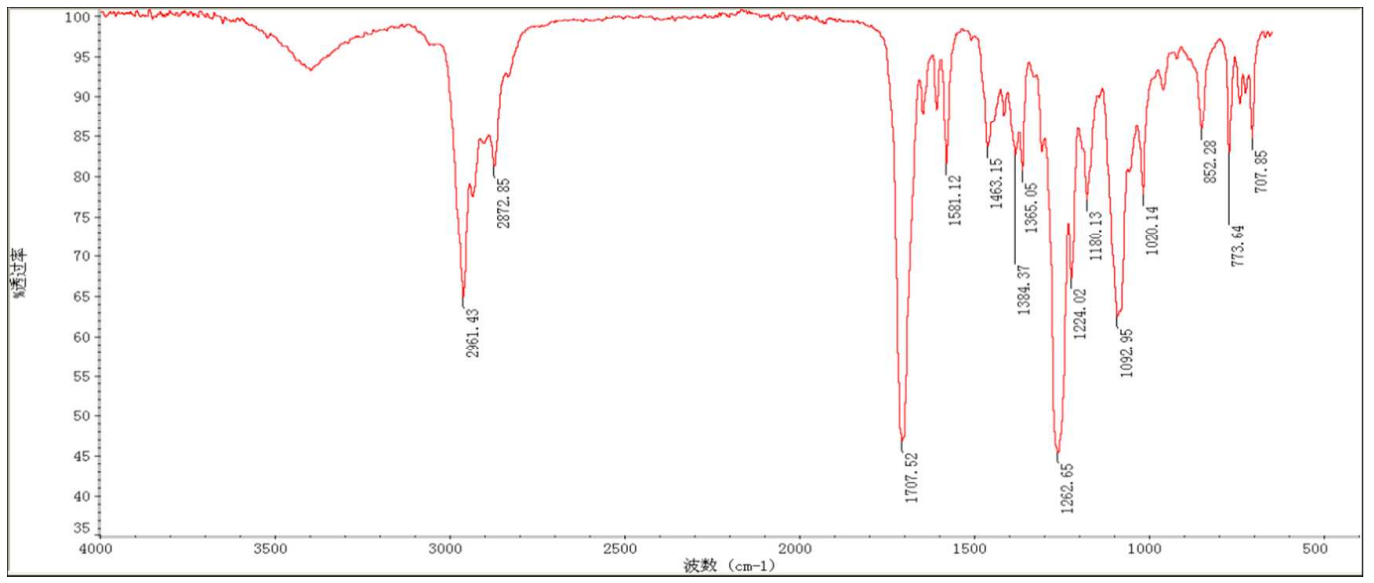

Figure S3. FT-IR spectrum of the compound 5c 


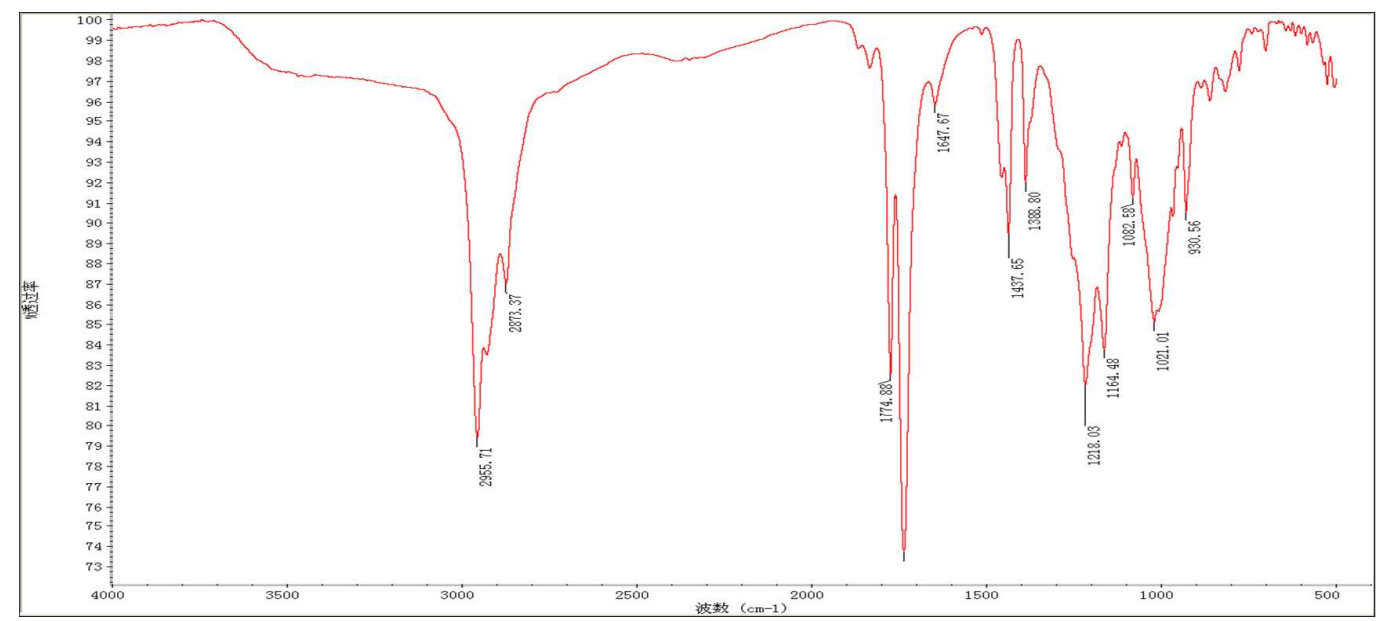

Figure S4. FT-IR spectrum of the compound 7a

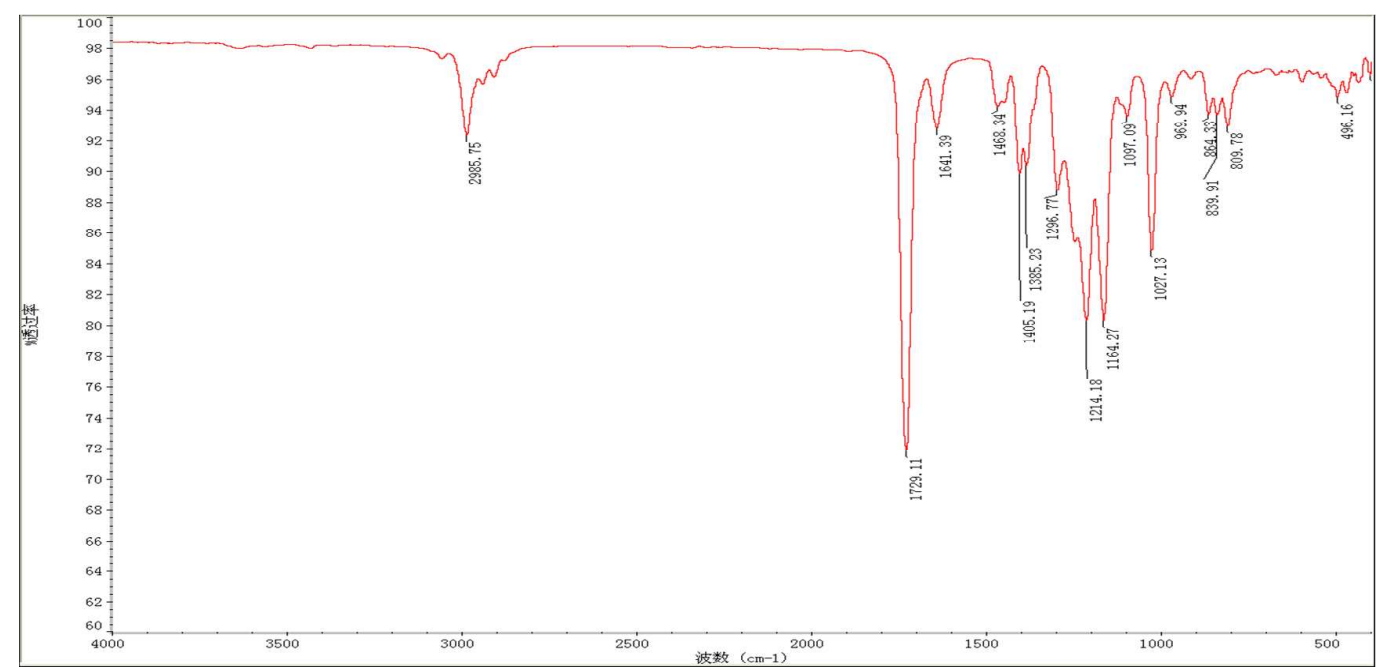

Figure S5. FT-IR spectrum of the compound 7b 




Figure S6. ${ }^{1} \mathrm{H}$ NMR spectrum of the compound $\mathbf{5 a}$

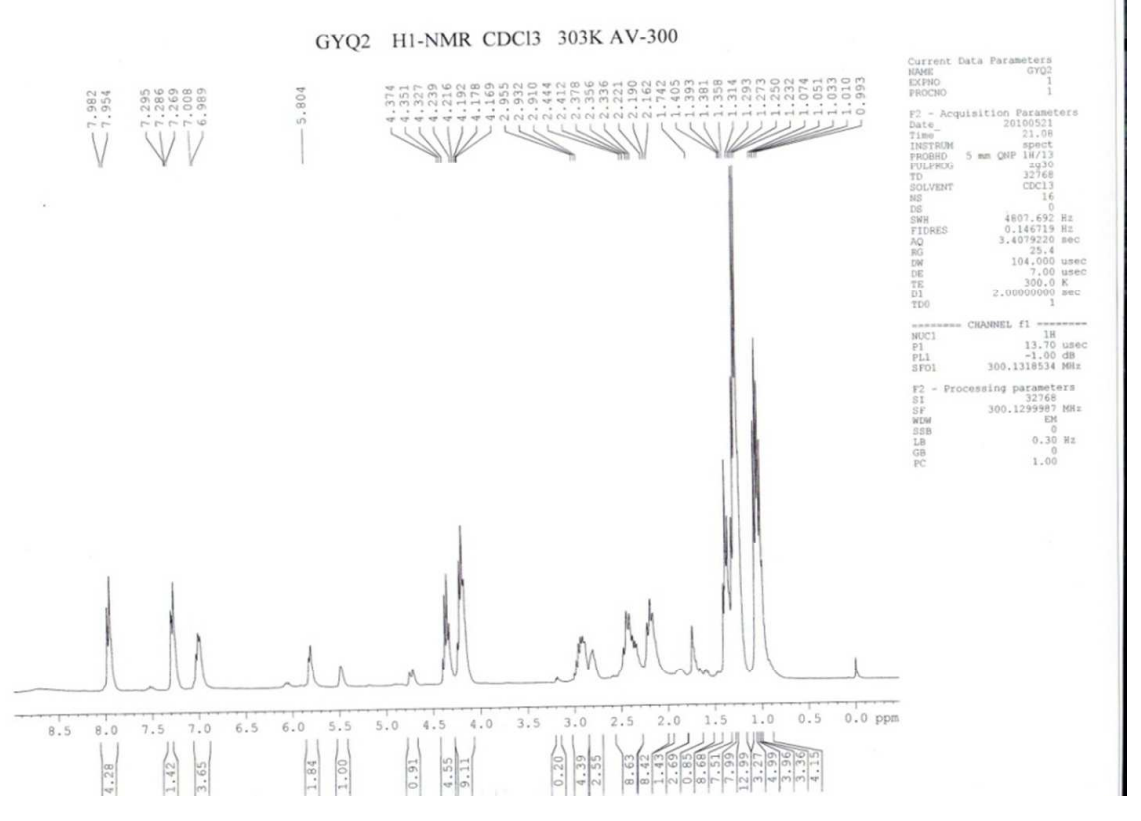

Figure S7. ${ }^{1} \mathrm{H}$ NMR spectrum of the compound $\mathbf{5 b}$ 


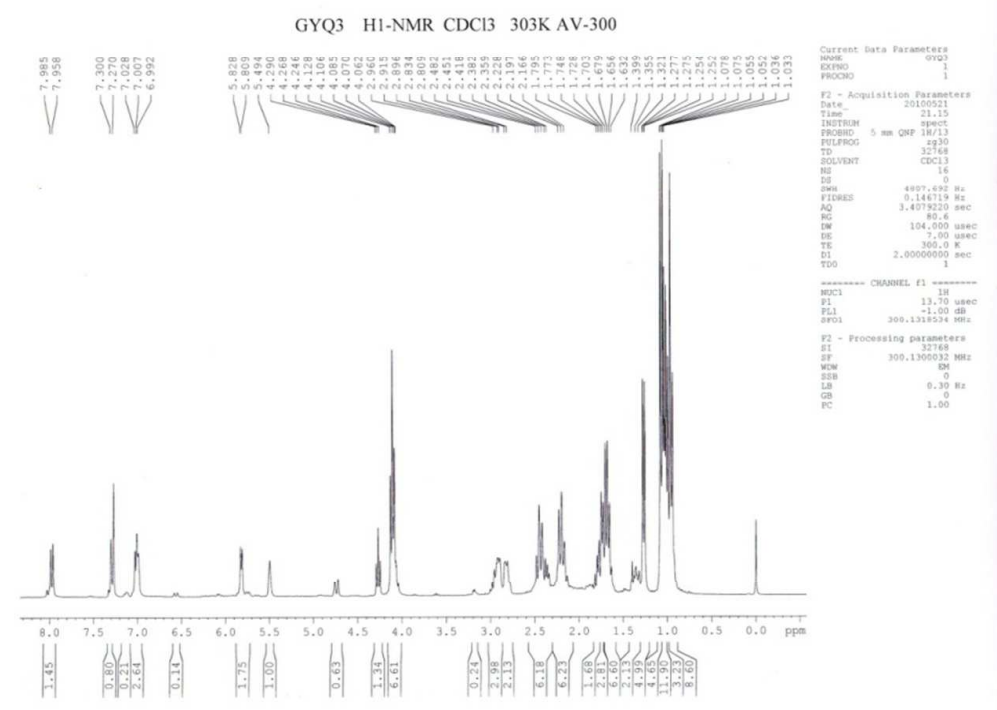

Figure S8. ${ }^{1} \mathrm{H}$ NMR spectrum of the compound $\mathbf{5 c}$



Figure S9. ${ }^{1} \mathrm{H}$ NMR spectrum of the compound 7a 


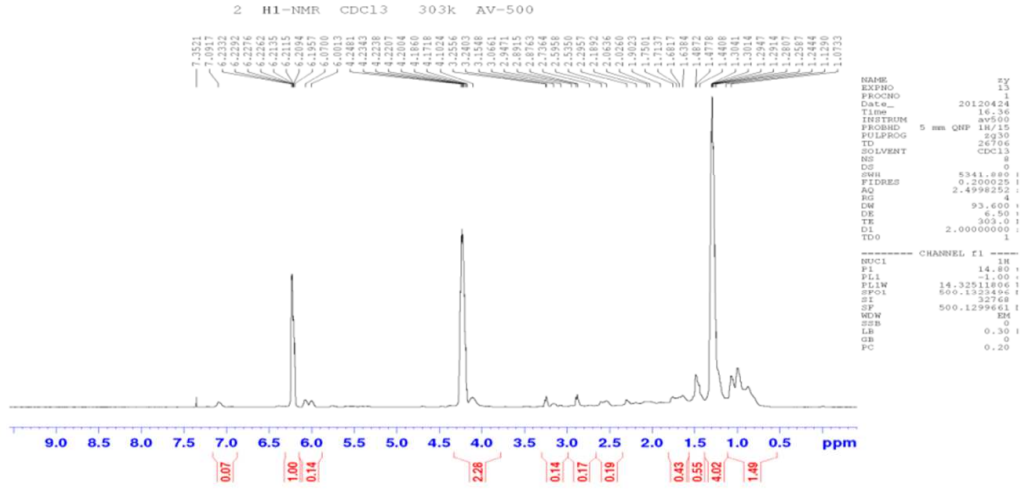

Figure S10. ${ }^{1}$ H NMR spectrum of the compound $\mathbf{7 b}$

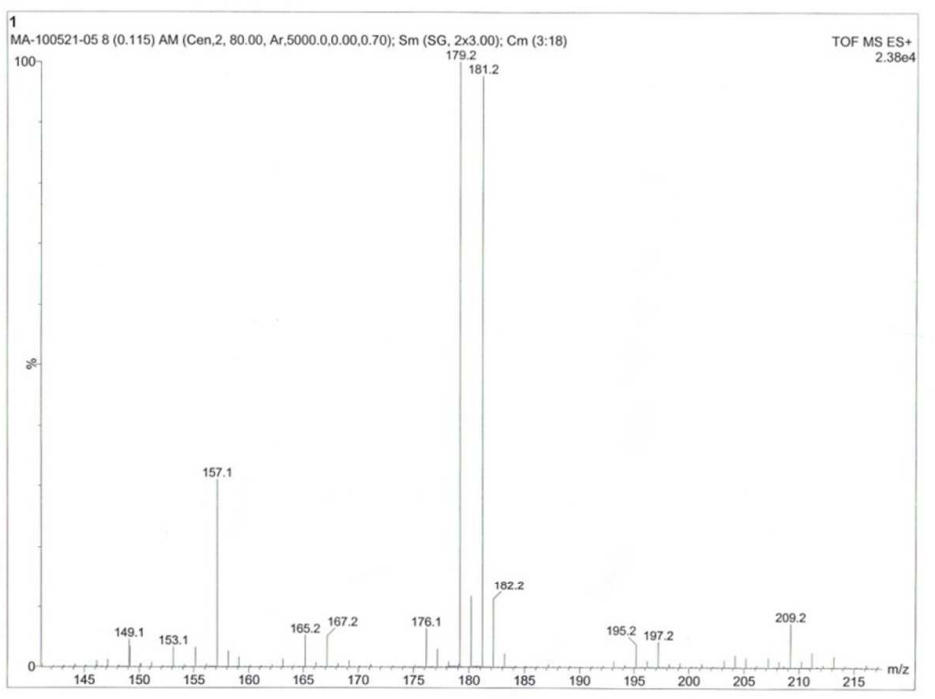

Figure S11. ESI-MS spectrum of the compound $\mathbf{5 a}$ 




Figure S12. ESI-MS spectrum of the compound $\mathbf{5 b}$

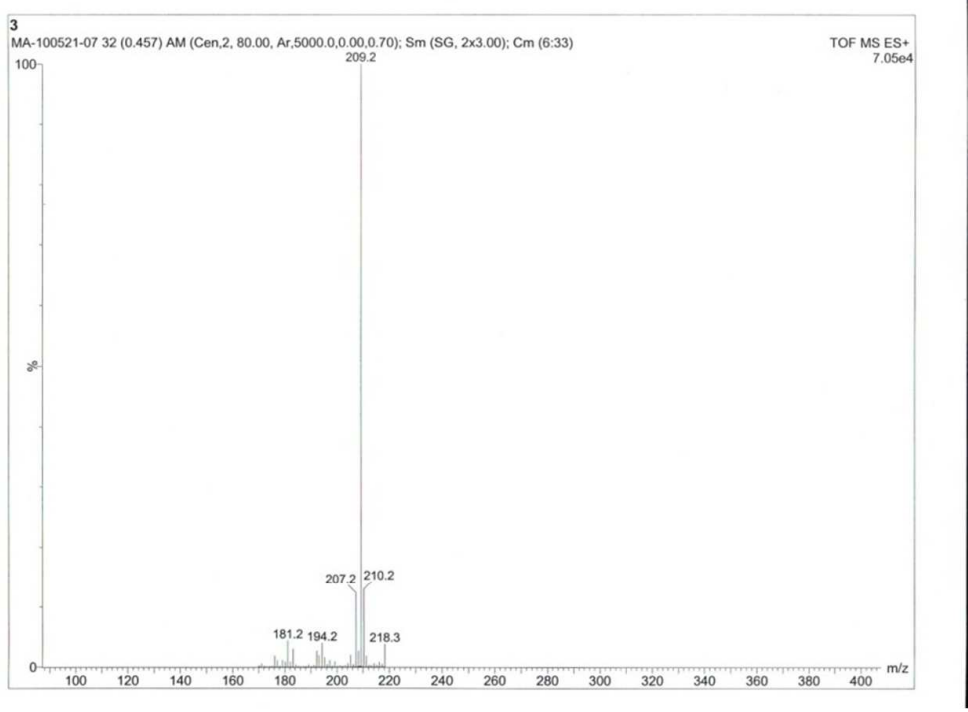

Figure S13. ESI-MS spectrum of the compound 5c 


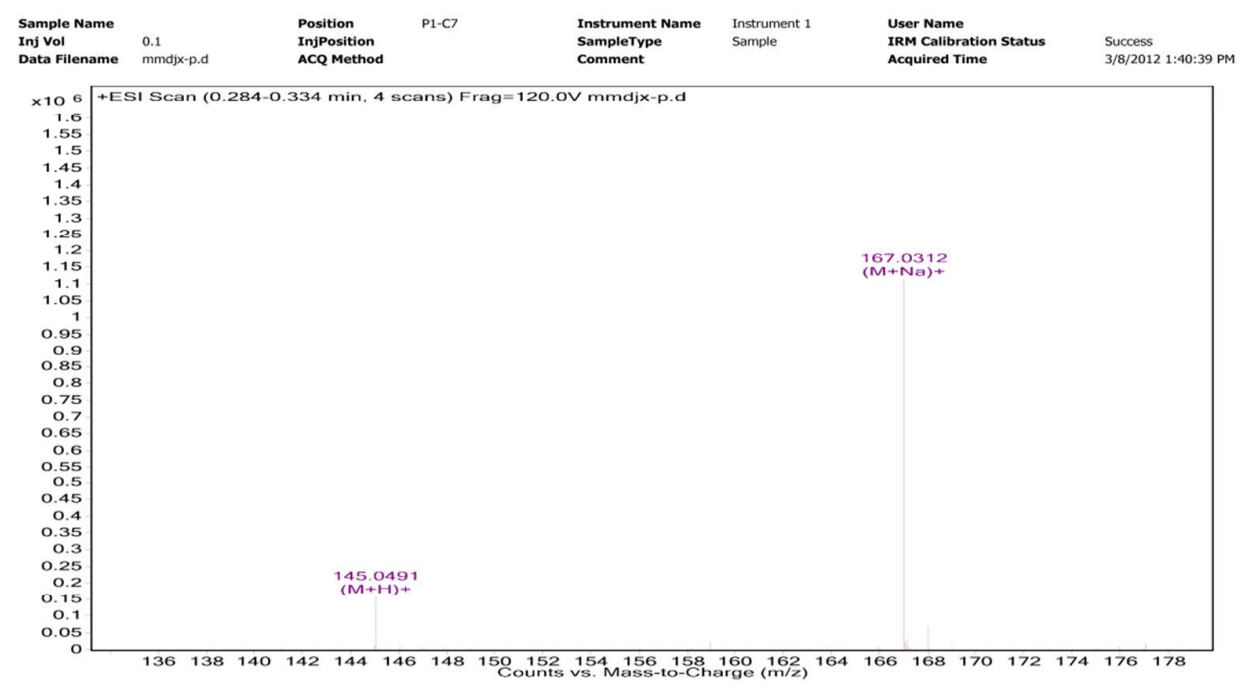

Figure S14. ESI-MS spectrum of the compound 7a

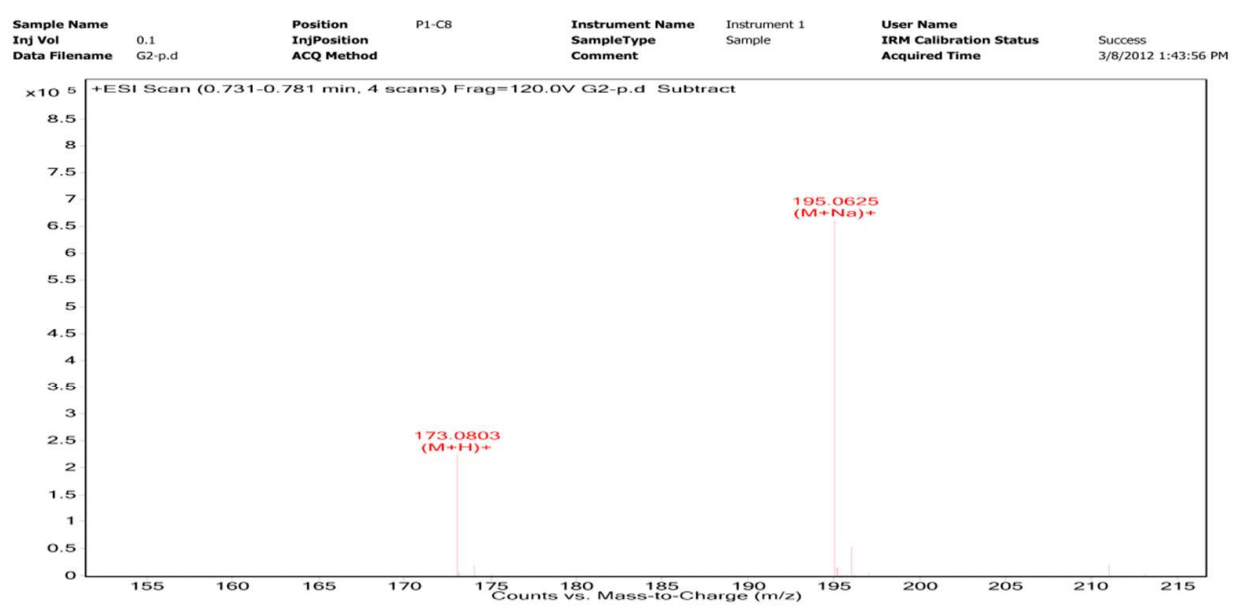

Figure S15. ESI-MS spectrum of the compound 7b 


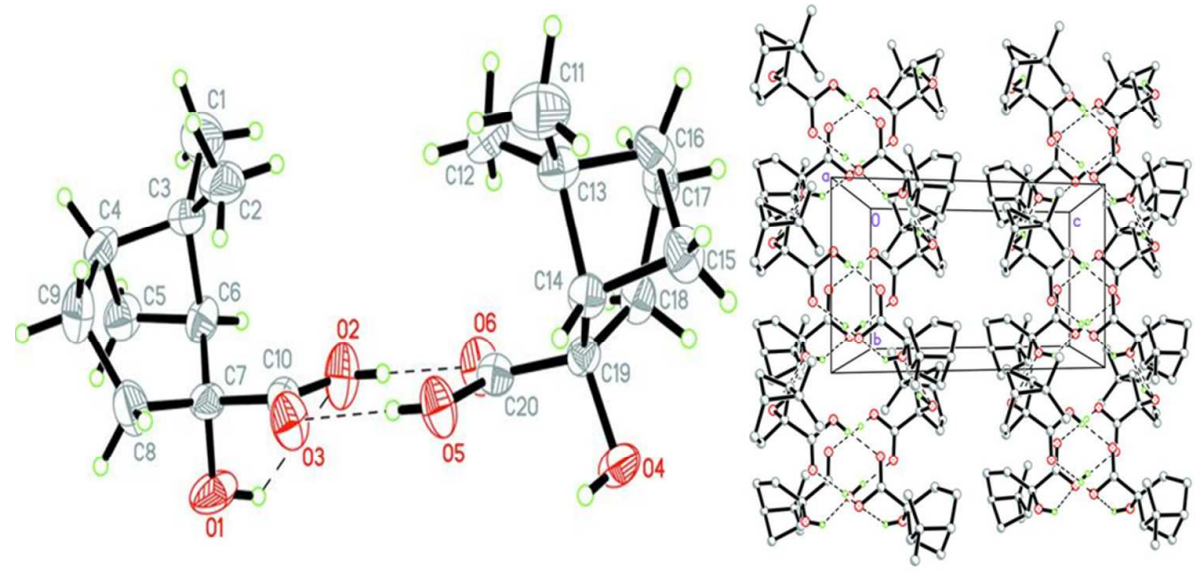

Figure S16. The asymmetric unit and crystal packing of compound 2.



Figure S17. The "breaking point" rule results. 

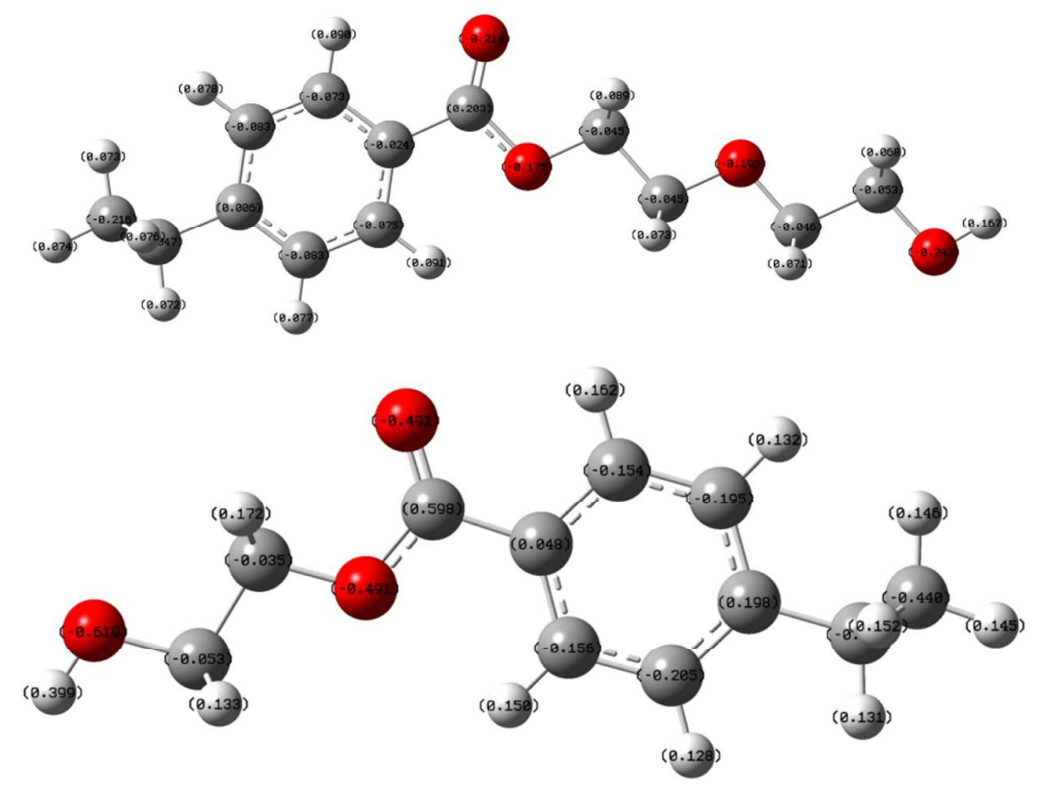

Figure S18. The charge distribution results of compounds $5 k$ and 51
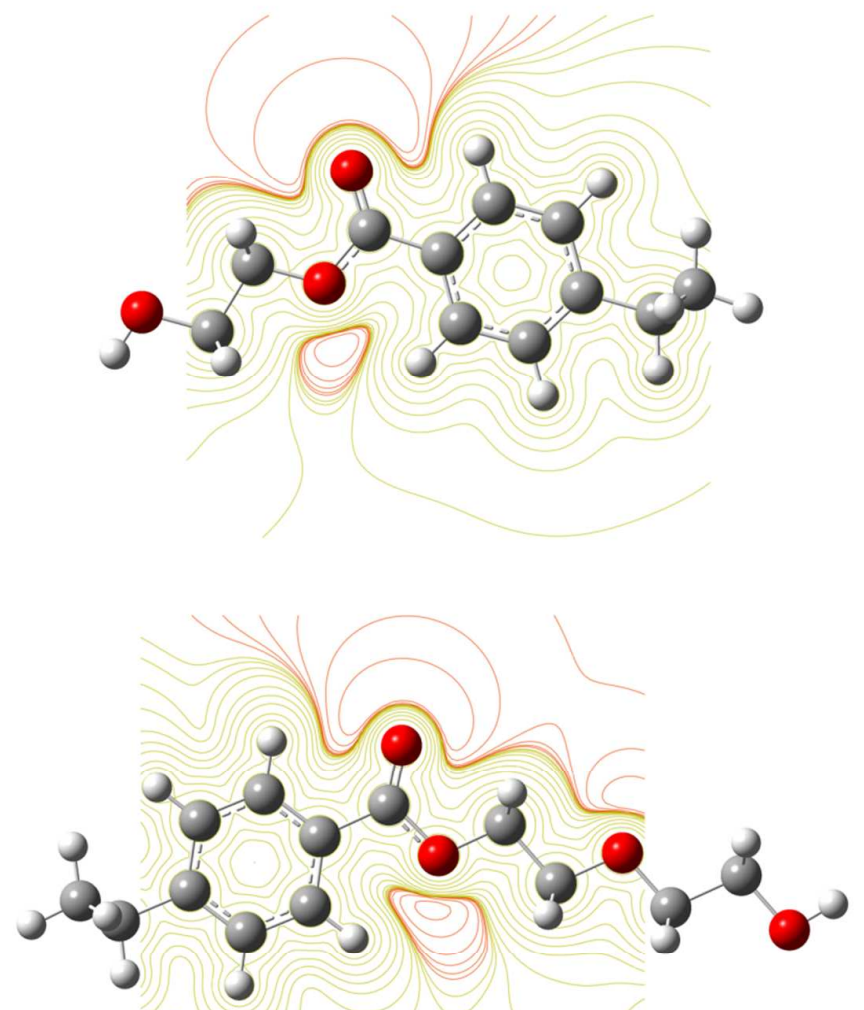

Figure S19. The electrostatic potential value results of title compounds $5 \mathrm{k}$ and 51 


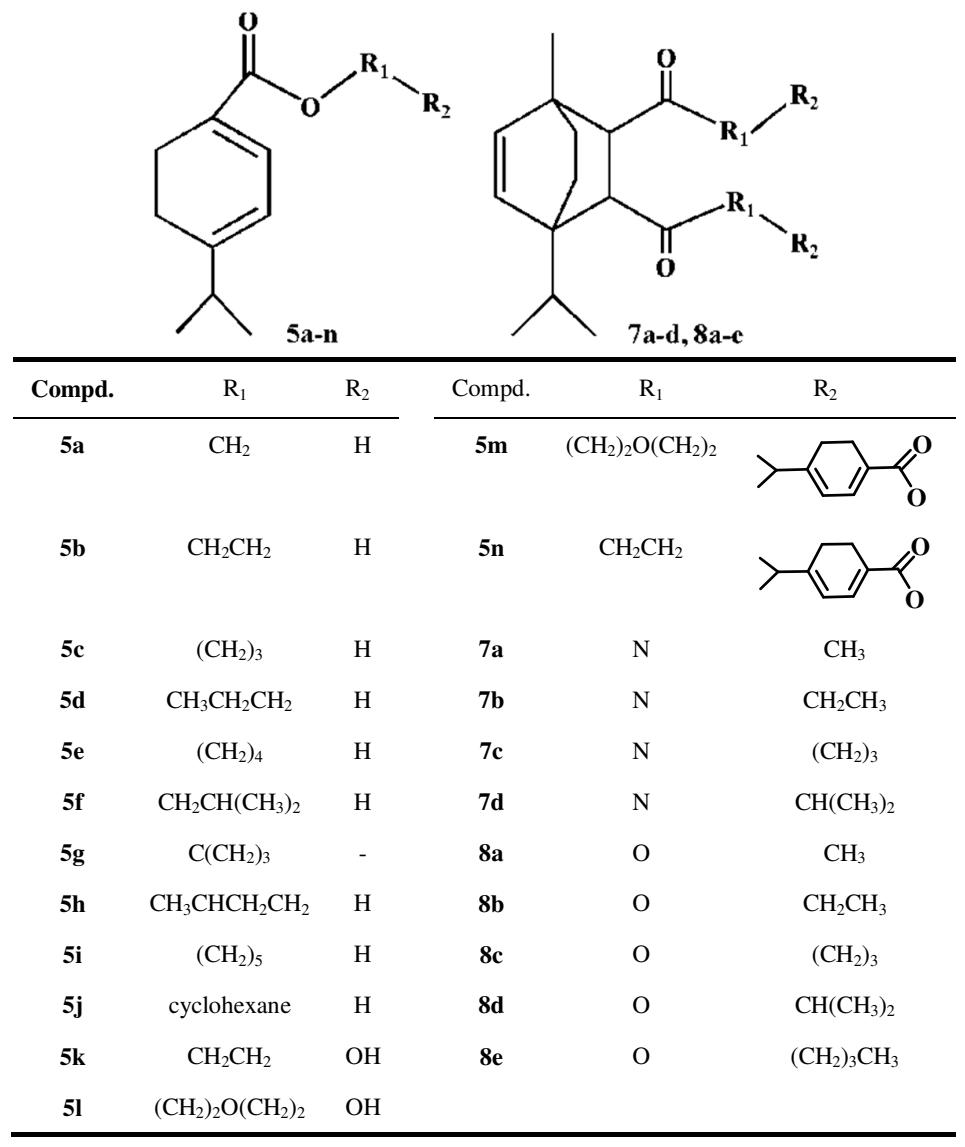

Table S1. The substituted groups of $\beta$-pinene derivatives

\begin{tabular}{|c|c|c|c|c|c|c|c|}
\hline \multirow[t]{2}{*}{ Compd. } & \multicolumn{3}{|c|}{$\begin{array}{l}\text { Fungicidal activity }(\%) \text { at } \\
\text { a concentration of }(\mu \mathrm{g} / \mathrm{mL})\end{array}$} & \multirow[t]{2}{*}{ Compd. } & \multicolumn{3}{|c|}{$\begin{array}{l}\text { Fungicidal activity }(\%) \text { at } \\
\text { a concentration of }(\mu \mathrm{g} / \mathrm{mL})\end{array}$} \\
\hline & 256 & 128 & 64 & & 256 & 128 & 64 \\
\hline 3 & 20 & 0 & - & $5 \mathrm{~m}$ & 38 & 0 & - \\
\hline $5 a$ & 20 & 5 & 0 & $5 n$ & 40 & 0 & - \\
\hline $5 \mathbf{b}$ & 20 & 5 & 0 & $7 a$ & 30 & 20 & 0 \\
\hline $5 c$ & 28 & 10 & 0 & $7 b$ & 30 & 20 & 0 \\
\hline 5d & 29 & 10 & 0 & $7 \mathrm{c}$ & 30 & 20 & 0 \\
\hline $5 e$ & 30 & 10 & 0 & $7 d$ & 30 & 20 & 0 \\
\hline $5 f$ & 30 & 10 & 0 & $8 a$ & 38 & 10 & 0 \\
\hline $5 g$ & 28 & 10 & 0 & $8 b$ & 35 & 10 & 0 \\
\hline $5 h$ & 29 & 10 & 0 & $8 c$ & 40 & 10 & 0 \\
\hline $5 \mathrm{i}$ & 20 & 5 & 0 & $8 d$ & 40 & 10 & 0 \\
\hline $5 j$ & 20 & 5 & 0 & $8 e$ & 40 & 10 & 0 \\
\hline $5 \mathbf{k}$ & 45 & 20 & 0 & Carbendazim & 100 & 100 & 90 \\
\hline 51 & 43 & 20 & 0 & & & & \\
\hline
\end{tabular}

Table S2. Fungicidal activity of compounds against $R$. solani 


\begin{tabular}{|c|c|c|c|c|c|c|c|}
\hline \multirow[t]{2}{*}{ Compd. } & \multicolumn{3}{|c|}{$\begin{array}{l}\text { Fungicidal activity }(\%) \text { at } \\
\text { a concentration of }(\mu \mathrm{g} / \mathrm{mL})\end{array}$} & \multirow[t]{2}{*}{ Compd. } & \multicolumn{3}{|c|}{$\begin{array}{l}\text { Fungicidal activity }(\%) \text { at } \\
\text { a concentration of }(\mu \mathrm{g} / \mathrm{mL})\end{array}$} \\
\hline & 256 & 128 & 64 & & 256 & 128 & 64 \\
\hline 3 & 10 & 0 & - & $5 \mathrm{~m}$ & 18 & 0 & - \\
\hline $5 a$ & 10 & 0 & - & $5 n$ & 10 & 0 & - \\
\hline $5 \mathbf{b}$ & 15 & 10 & 0 & $7 a$ & 30 & 20 & 0 \\
\hline $5 \mathrm{c}$ & 18 & 10 & 0 & $7 b$ & 30 & 20 & 0 \\
\hline $5 d$ & 15 & 10 & 0 & $7 c$ & 30 & 20 & 0 \\
\hline $5 e$ & 20 & 10 & 0 & $7 d$ & 30 & 20 & 0 \\
\hline $5 f$ & 20 & 10 & 0 & $8 \mathbf{a}$ & 28 & 10 & 0 \\
\hline $5 \mathrm{~g}$ & 19 & 10 & 0 & $8 b$ & 25 & 10 & 0 \\
\hline $5 \mathrm{~h}$ & 19 & 10 & 0 & $8 c$ & 30 & 10 & 0 \\
\hline $5 i$ & 10 & 0 & - & $8 d$ & 30 & 10 & 0 \\
\hline $5 j$ & 10 & 0 & - & $8 e$ & 30 & 10 & 0 \\
\hline $5 \mathbf{k}$ & 35 & 20 & 0 & Carbendazim & 100 & 100 & 95 \\
\hline 51 & 35 & 20 & 0 & & & & \\
\hline
\end{tabular}

Table S3. Fungicidal activity of compounds against $F$. graminearum 


\begin{tabular}{|c|c|c|c|c|c|c|}
\hline \multirow[b]{2}{*}{ No. } & \multirow[b]{2}{*}{ Compd. } & \multirow[b]{2}{*}{$\log \mathrm{IC}_{50}$} & \multicolumn{4}{|c|}{ Structure descriptor } \\
\hline & & & $\mathrm{DM}^{\mathrm{a}}$ & $\mathrm{MV}^{\mathrm{b}}$ & $\mathrm{NOEL}^{\mathrm{c}}$ & $\mathrm{TDM}^{\mathrm{d}}$ \\
\hline 1 & 3 & 2.131 & 0.3528 & 1.9154 & 32.0000 & 2.8430 \\
\hline 2 & $5 a$ & 1.858 & 0.3463 & 1.9142 & 35.0000 & 2.4140 \\
\hline 3 & $5 b$ & 1.816 & 0.3493 & 1.9141 & 38.0000 & 2.2530 \\
\hline 4 & $5 \mathrm{c}$ & 1.639 & 0.3559 & 1.9136 & 41.0000 & 2.0570 \\
\hline 5 & $5 d$ & 1.734 & 0.3559 & 1.9136 & 41.0000 & 2.0570 \\
\hline 6 & $5 e$ & 1.516 & 0.3488 & 1.9141 & 44.0000 & 2.2990 \\
\hline 7 & $5 f$ & 1.593 & 0.3483 & 1.9141 & 44.0000 & 2.2660 \\
\hline 8 & $5 \mathrm{~g}$ & 1.643 & 0.3589 & 1.9136 & 44.0000 & 2.1090 \\
\hline 9 & $5 \mathbf{h}$ & 1.618 & 0.3556 & 1.9136 & 44.0000 & 2.0880 \\
\hline 10 & $5 i$ & 1.798 & 0.3488 & 1.9141 & 47.0000 & 2.3080 \\
\hline 11 & $5 \mathbf{j}$ & 1.890 & 0.3513 & 1.9141 & 49.0000 & 2.2170 \\
\hline 12 & $5 \mathbf{k}$ & 0.780 & 0.3519 & 1.9751 & 41.0000 & 3.9580 \\
\hline 13 & 51 & 0.820 & 0.3522 & 1.9757 & 50.0000 & 2.3360 \\
\hline 14 & $5 \mathrm{~m}$ & 2.124 & 0.3521 & 1.9527 & 78.0000 & 4.2030 \\
\hline 15 & $5 n$ & 2.113 & 0.3481 & 1.9133 & 69.0000 & 0.0720 \\
\hline 16 & $7 a$ & 1.466 & 0.3149 & 1.9173 & 56.0000 & 6.1750 \\
\hline 17 & $7 b$ & 1.430 & 0.3138 & 1.9176 & 62.0000 & 6.0240 \\
\hline 18 & $7 \mathrm{c}$ & 1.357 & 0.3134 & 1.9177 & 68.0000 & 5.9450 \\
\hline 19 & $7 d$ & 1.387 & 0.3182 & 1.9174 & 68.0000 & 5.9190 \\
\hline 20 & $8 a$ & 1.342 & 0.3046 & 1.9147 & 56.0000 & 3.4220 \\
\hline 21 & $8 b$ & 1.263 & 0.3071 & 1.9148 & 62.0000 & 3.6340 \\
\hline 22 & $8 \mathrm{c}$ & 1.157 & 0.3073 & 1.9148 & 68.0000 & 3.6410 \\
\hline 23 & $8 d$ & 1.192 & 0.3124 & 1.9147 & 68.0000 & 3.4360 \\
\hline 24 & $8 \mathrm{e}$ & 1.112 & 0.3073 & 1.9148 & 74.0000 & 3.6760 \\
\hline
\end{tabular}

Table S4. Fungicidal activity and structure descriptors of the title compounds 


\begin{tabular}{|c|c|c|c|c|}
\hline No. & Compd. & Calc. $\log \mathrm{IC}_{50}$ & Exp. $\log \mathrm{IC}_{50}$ & Difference \\
\hline 1 & 3 & 2.1477 & 2.1310 & 0.0167 \\
\hline 2 & $5 a$ & 1.8175 & 1.8600 & -0.0425 \\
\hline 3 & $5 b$ & 1.7400 & 1.8160 & -0.0760 \\
\hline 4 & $5 \mathrm{c}$ & 1.7706 & 1.6390 & 0.1316 \\
\hline 5 & $5 d$ & 1.7706 & 1.7340 & 0.0366 \\
\hline 6 & $5 e$ & 1.5776 & 1.5160 & 0.0616 \\
\hline 7 & $5 f$ & 1.5626 & 1.5950 & -0.0324 \\
\hline 8 & $5 \mathrm{~g}$ & 1.7735 & 1.6450 & 0.1285 \\
\hline 9 & $5 \mathrm{~h}$ & 1.7007 & 1.6150 & 0.0857 \\
\hline 10 & $5 \mathbf{i}$ & 1.5280 & 1.8000 & -0.2720 \\
\hline 11 & $5 j$ & 1.7056 & 1.8900 & -0.1844 \\
\hline 12 & $5 \mathrm{k}$ & 0.8994 & 0.7810 & 0.1184 \\
\hline 13 & 51 & 0.7519 & 0.8040 & -0.0521 \\
\hline 14 & $5 \mathrm{~m}$ & 2.0210 & 2.1240 & -0.1030 \\
\hline 15 & $5 n$ & 2.2027 & 2.1130 & 0.0897 \\
\hline 16 & $7 \mathbf{a}$ & 1.4921 & 1.4460 & 0.0461 \\
\hline 17 & $7 b$ & 1.3887 & 1.4300 & -0.0413 \\
\hline 18 & $7 c$ & 1.3454 & 1.3570 & -0.0116 \\
\hline 19 & $7 d$ & 1.4482 & 1.3870 & 0.0612 \\
\hline 20 & $8 \mathbf{a}$ & 1.2487 & 1.3420 & -0.0933 \\
\hline 21 & $8 b$ & 1.2365 & 1.2630 & -0.0265 \\
\hline 22 & $8 \mathrm{c}$ & 1.1872 & 1.1570 & 0.0302 \\
\hline 23 & $8 d$ & 1.2677 & 1.1920 & 0.0757 \\
\hline 24 & $8 \mathrm{e}$ & 1.1648 & 1.1120 & 0.0528 \\
\hline
\end{tabular}

Table S5. The difference between the experimental $\log \mathrm{IC}_{50}$ and predicted $\log \mathrm{IC}_{50}$

\begin{tabular}{cccccccccc}
\hline Training set & $N$ & $R^{2}$ & $F$ & $S^{2}$ & Test set & $N$ & $R^{2}$ & $F$ & $S^{2}$ \\
\hline A+B & 16 & 0.9500 & 47.78 & 0.0130 & C & 8 & 0.9495 & 47.90 & 0.0131 \\
B+C & 16 & 0.9511 & 48.58 & 0.0121 & A & 8 & 0.9514 & 48.25 & 0.0128 \\
A+C & 16 & 0.9524 & 48.63 & 0.0129 & B & 8 & 0.9593 & 48.47 & 0.0133 \\
Average & & 0.9512 & 48.33 & 0.0127 & Average & & 0.9534 & 48.21 & 0.0131 \\
\hline
\end{tabular}

Table S6. Internal validation of the QSAR model 


\begin{tabular}{|c|c|c|c|}
\hline Compds & $\begin{array}{l}\text { Energy value } \\
\text { (a.u.) }\end{array}$ & Compds & $\begin{array}{l}\text { Energy value } \\
\text { (a.u.) }\end{array}$ \\
\hline 3 & -538.6220 & $5 \mathbf{l}$ & -835.6393 \\
\hline $5 \mathbf{a}$ & -578.0739 & $5 \mathrm{~m}$ & -1301.2271 \\
\hline $5 \mathbf{b}$ & -617.3928 & $5 n$ & -1154.9158 \\
\hline $5 c$ & -656.7065 & $7 a$ & -885.5024 \\
\hline $5 d$ & -656.7073 & $7 b$ & -963.9335 \\
\hline $5 e$ & -696.0202 & $7 c$ & -1042.5613 \\
\hline $5 f$ & -696.0211 & $7 d$ & -1042.5635 \\
\hline $5 g$ & -696.0244 & $\mathbf{8 a}$ & -924.7643 \\
\hline $5 \mathrm{~h}$ & -696.0208 & $\mathbf{8 b}$ & -1003.3840 \\
\hline $5 \mathbf{i}$ & -735.3339 & $8 c$ & -1081.9921 \\
\hline $5 \mathbf{j}$ & -733.4480 & $\mathbf{8 d}$ & -1082.0011 \\
\hline $5 \mathbf{k}$ & -692.5936 & $8 \mathrm{e}$ & -1160.6000 \\
\hline
\end{tabular}

Table S7. The calculated energy value of title compounds. 